\title{
Seleção de procedências e progênies de Araucaria angustifolia (Bert.) O. Kuntze para produção de madeira e pinhão
}

\author{
Selection of provenances and progenies of Araucaria angustifolia (Bert.) \\ O. Kuntze for wood and seed production
}

\section{Janaina Rodrigues da Silva', Wanderley dos Santos ${ }^{1}$, Mario Luiz Teixeira de Moraes ${ }^{1}$, Jarbas Yukio Shimizu², Valderes Aparecida de Sousa² e Ananda Virginia de Aguiar ${ }^{2}$}

\section{RESUMO}

Foi efetuada a caracterização genética e estimado o ganho genético com a seleção em um teste de procedências e progênies de Araucaria angustifólia, visando a produção de madeira e sementes. $O$ teste foi estabelecido em 1980 em Colombo-PR, no delineamento de blocos de famílias compactadas, no espaçamento de 3,0 x 2,0 m, com duas repetições, 12 procedências, 110 progênies e dez plantas por parcela. Os caracteres avaliados foram: DAP (diâmetro a altura do peito), ALT (altura total), VOL (volume cilíndrico), DMC (diâmetro médio de copa), ESP (espessura da casca) e os caracteres de produção de sementes (pinhão). As estimativas dos parâmetros genéticos e o ganho por seleção foram estimados com base no método REML/BLUP. Foi detectada variação genética significativa entre procedências para ALT, VOL, DMC e ESP e entre progênies para VOL. O coeficiente de variação genética individual $\left(C V_{g i}\right)$ foi alto para DAP e VOL $(\geq 10,2 \%)$ e a herdabilidade individual no sentido restrito $\left(\hat{h}_{a}^{2}\right)$ variou de baixa $(0,03)$ para DMC a alta magnitude $(0,60)$ para VOL. A estratégia de seleção de menor intensidade $(38 \%)$ com base no DAP aos 32 anos foi considerada a mais recomendada, por manter o maior nível de diversidade genética $(7,6 \%)$ e tamanho efetivo populacional (55). As procedências de Irati-PR e Itapeva-SP devem ser priorizadas nos próximos ciclos de melhoramento da espécie por apresentarem melhor desempenho em crescimento.

Palavras-chave: pinheiro do paraná; caracteres quantitativos; melhoramento genético; ganho genético por seleção.

\begin{abstract}
The genetic characterization and the prediction of genetic gain in a test of provenances and progenies of Araucaria angustifolia, was carried out aiming wood and seeds production. The test was established in 1980 in the block randomized design of compacted families, spacing $3.0 \times 2.0 \mathrm{~m}$, with 2 replicates, 12 provenances, 110 progenies and ten plants per plot. The evaluated traits were: $\mathrm{DBH}$ (diameter at breast height), ALT (total height), VOL (cylindrical volume), DMC (average crown diameter), ESP (bark thickness) and seed (pinhão) production traits. Estimates of the genetic parameters and the gain by selection were estimated using the REML/BLUP method. Significant genetic variation was observed between provenances for ALT, VOL, DMC e ESP, and progenies for VOL. The coefficient of individual genetic variation $\left(C_{g i}\right)$ was higher for $\mathrm{DBH}$ and $\mathrm{VOL}(\geq 10.2 \%)$. Moreover, narrow-sense individual heritability $\left(\hat{h}_{a}^{2}\right)$ varied from low $(0.03)$ DMC to high magnitude (0.60) VOL. The strategy of selection of lower intensity (38\%) based on DAP at age 32 was considered the most recommended because it maintains the highest level of genetic diversity $(7.6 \%)$ and effective population size (55). The Irati-PR and Itapeva- SP provencances should be prioritized in the next cycles of breeding of the species since those presente better growth performance.
\end{abstract}

Keywords: Pinheiro do paraná; quantitative trait; genetic improvement; genetic gain.

\section{INTRODUÇÃO}

A Araucaria angustifolia (Bert.) O. Kuntze, conhecida popularmente como pinheiro-do-paraná ou araucária, é uma espécie representativa da Floresta Ombrófila Mista, considerada uma unidade fitogeográfica do bioma Mata Atlântica (KUHN; ERNESTO; MARIATH, 2014). Durante décadas, a

1. Faculdade de Engenharia de Ilha Solteira - FEIS, Universidade Estadual Paulista "Júlio de Mesquita Filho" - Unesp. Ilha Solteira / SP, Brasil. E-mail: janainars t@hotmail.com

2. Empresa Brasileira de Pesquisa Agropecuária. Embrapa Florestas - Embrapa. Colombo / PR, Brasil.

Sci. For., Piracicaba, v. 46, n. 120, p. 519-531, dez. 2018 DOI: dx.doi.org/10.18671/scifor.v46n120.01 
A. angustiolia foi a espécie mais importante economicamente para o Brasil. Sua exploração desenfreada entre os anos de 1930 a 1950 para uso da madeira, bem como a expansão das áreas agrícolas, fizeram com que as áreas de florestas com araucária fossem reduzidas drasticamente. Da imensa área ocupada por esse tipo de vegetação, restaram apenas de 1 a 2\% da vegetação original (GUERRA et al., 2002). Os remanescentes isolados geograficamente (ANSELMINI; ZANETTE, 2011) apresentam variados tamanhos, quase sempre em formações mistas, com espécies folhosas e densos sub-bosques que dificultam a regeneração natural da araucária (SHIMIZU; JAEGER, SOPCHAKI, 2000). Fatores como a exploração sem a devida reposição, a falta de incentivo à produção e o manejo da araucária contribuíram para a espécie ser incluída na lista das espécies ameaçadas de extinção (THOMAS, 2013).

A espécie é uma gimnosperma, apresenta árvores com 10 a $35 \mathrm{~m}$ de altura e 50 a $120 \mathrm{~cm}$ de diâmetro à altura do peito, atingindo excepcionalmente $50 \mathrm{~m}$ de altura e $250 \mathrm{~cm}$ ou mais de DAP, na idade adulta (CARVALHO, 2002). O tronco é cilíndrico, reto e raramente ramificado em dois ou mais, com casca grossa e resinosa (REITZ et al., 1983). O crescimento inicial é lento, porém a partir do terceiro ano em sítios adequados apresenta incremento médio anual em altura de $1 \mathrm{~m}$, e a partir do quinto ano, incremento médio em DAP de 1,5 a 2,0 cm (CARVALHO, 1994). É uma espécie dioica, apresentando indivíduos do sexo masculino e feminino, sendo que a distinção entre eles é feita com base no formato das estruturas reprodutivas, as quais são cilíndricas nos indivíduos masculinos e esféricas nos femininos (FONTES; DAVIDE; DAVIDE, 2001). A polinização é cruzada do tipo anemófila. Após a polinização, ocorre o desenvolvimento dos pinhões que amadurecem de 20 a 24 meses mais tarde (ZANETTE et al., 2017).

A A. angustifolia destaca-se por apresentar madeira de alta qualidade, com boas características físicas e mecânicas e de amplo uso, e como principal produto não madeireiro, o pinhão (semente), com alto valor nutricional (HESS et al., 2010), rico em reservas energéticas tanto para o homem quanto para os animais silvestres, consumido mais em nível regional. Apesar da grande importância econômica, social e ecológica da espécie, são poucos os incentivos para o seu plantio. No ano de 2015 a área plantada com a espécie atingiu 11.038 ha (IBÁ, 2016). Ainda que a produtividade de madeira da araucária seja inferior às espécies do gênero Pinus e Eucalyptus, deve-se considerar as possibilidades de usos múltiplos e os benefícios ambientais do cultivo florestal dessa espécie (MARTINS et al., 2017). Segundo Shimizu et al. (1999), o estabelecimento de reflorestamentos atrelados aos planos de manejo, utilizando-se sementes de procedências conhecidas e de maior produtividade podem fornecer madeira para fins comerciais, proporcionar cobertura florestal e proteção ambiental, atender a demanda de pinhões para consumo humano e o estabelecimento de novos povoamentos produtivos. Para tanto, são necessárias pesquisas que auxiliem no fornecimento de informações sobre o crescimento, a biologia reprodutiva, a produção e o aproveitamento da araucária. Essas informações podem aumentar o interesse da utilização da espécie em plantios comerciais visando à produção de sementes e madeira de maneira sustentável, reduzindo a pressão sobre os remanescentes nativos.

O conhecimento do padrão de crescimento da espécie pode trazer benefícios econômicos, ambientais e sociais, acompanhando e predizendo as mudanças na floresta, por meio das técnicas de manejo (HESS; SCHNEIDER.; FINGER, 2009). Nesse contexto, a identificação de árvores com características fenotípicas desejáveis é de extrema importância. Essas podem ser selecionadas ao longo do tempo e incorporadas aos programas de melhoramento genético, visando a obtenção de genótipos mais produtivos (GARTLAND et al., 2003). O objetivo do presente estudo foi caracterizar geneticamente uma população de A. angustifolia instalada em Colombo-PR, visando a produção de madeira e sementes. Para tanto, foram estimados os parâmetros genéticos para os caracteres quantitativos, a avaliação da produção de sementes e a seleção de árvores superiores. Essas informações propiciarão a ampliação do conhecimento sobre a capacidade produtiva de algumas procedências e progênies da espécie, além de viabilizar a seleção das matrizes de alto valor genético, com o propósito de formar pomares de sementes clonais e estabelecer testes de progênies de segunda geração. Isso possibilitará plantios com maior capacidade de produção.

\section{MATERIAL E MÉTODOS}

\section{Amostragem e delineamento experimental}

O estudo foi realizado em um teste de procedências e progênies de A. angustifolia estabelecido no munícipio de Colombo-PR ( $25^{\circ} 19^{\prime \prime}$ S, $\left.25^{\circ} 19^{\prime \prime} \mathrm{W}\right)$, com altitude de $950 \mathrm{~m}$, temperatura média de $16,6^{\circ} \mathrm{C}$ e precipitação média de $1596 \mathrm{~mm}$. O clima da região, segundo a classificação de Köppen, 
é do tipo $\mathrm{Cfb}$ caracterizado como temperado. O solo é classificado como cambissolo distrófico saprolito, de textura média, com baixa fertilidade (EMBRAPA, 2013). O teste foi implantado em 1980, com base em sementes coletadas em povoamentos naturais localizados nas Regiões Sul e Sudeste do Brasil (Tabela 1). O delineamento experimental utilizado foi o de blocos de famílias compactadas, com parcelas subdividas, com duas repetições, 12 procedências, 110 progênies e dez plantas por parcela no espaçamento 3,0 x 2,0 m. Após o desbaste seletivo realizado no ano de 2001, com base no volume de madeira, permaneceram na área 210 indivíduos com aproximadamente uma árvore x progênie x bloco. Os caracteres silviculturais foram avaliados entre os anos de 2010 a 2013, sendo estes: i) diâmetro a altura do peito (DAP, cm); ii) altura total (ALT, m); iii) diâmetro médio de copa (DMC, m); iv) espessura da casca (ESP, $\mathrm{cm}$ ) e volume cilíndrico (VOL, $\mathrm{m}^{3}$.arv ${ }^{-1}$ ), o qual foi calculado conforme a expressão:

Tabela 1. Número de progênies, coordenadas geográficas, altitude e características climáticas dos locais de origem das procedências de $A$. angustifolia do teste de procedências e progênies implantado em Colombo-PR, em 1980.

Table 1. Number of progenies, geographic coordinates, altitude and climate characteristics of the places of origin of the $A$. angustifolia provenances of provenances and progenies test established in Colombo municipality, Paraná State in 1980.

\begin{tabular}{|c|c|c|c|c|c|c|c|}
\hline Procedência & $\begin{array}{c}\mathrm{N}^{0} \text { de } \\
\text { progênies }\end{array}$ & $\begin{array}{l}\text { Lat. } \\
\text { (S) }\end{array}$ & $\begin{array}{l}\text { Long. } \\
\text { (W) }\end{array}$ & $\begin{array}{l}\text { Alt. } \\
\text { (m) }\end{array}$ & $\begin{array}{l}\text { Prec. } \\
(\mathrm{mm})\end{array}$ & $\begin{array}{c}\text { Temp. } \\
\text { Média }\left({ }^{\circ} \mathrm{C}\right)\end{array}$ & Clima \\
\hline Barbacena, MG & 7 & $21^{\circ} 22^{\prime} 64^{\prime \prime}$ & $43^{\circ} 77^{\prime} 42^{\prime \prime}$ & 1205 & 1482 & $18,8^{\circ}$ & Cwb \\
\hline Caçador, SC & 9 & $26^{\circ} 46^{\prime} 00^{\prime \prime}$ & $51^{\circ} 01^{\prime} 00^{\prime \prime}$ & 960 & 1707 & $16,3^{\circ}$ & $\mathrm{Cfb}$ \\
\hline Campos do Jordão, SP & 10 & $22^{\circ} 45^{\prime} 00^{\prime \prime}$ & $45^{\circ} 30^{\prime} 00^{\prime \prime}$ & 1800 & 1797 & $13,6^{\circ}$ & $\mathrm{Cfb}$ \\
\hline Chapecó, SC & 7 & $27^{\circ} 07^{\prime} 00^{\prime \prime}$ & $52^{\circ} 36^{\prime} 00^{\prime \prime}$ & 675 & 1997 & $18,9^{\circ}$ & Cfa \\
\hline Congonhal, MG & 6 & $22^{\circ} 07^{\prime} 52^{\prime \prime}$ & $46^{\circ} 02^{\prime} 37^{\prime \prime}$ & 854 & 1443 & $19,9^{\circ}$ & Cfa \\
\hline Ipuiúna de Caldas, MG & 14 & $22^{\circ} 09^{\prime} 91^{\prime \prime}$ & $46^{\circ} 17^{\prime} 69^{\prime \prime}$ & 1300 & 1605 & $18,3^{\circ}$ & Cwb \\
\hline Irati, PR & 8 & $25^{\circ} 30^{\prime} 00^{\prime \prime}$ & $50^{\circ} 36^{\prime} 00^{\prime \prime}$ & 880 & 1476 & $17,5^{\circ}$ & Cfb \\
\hline Irati (tardio), $\mathrm{PR}^{*}$ & 10 & $25^{\circ} 30^{\prime} 00^{\prime \prime}$ & $50^{\circ} 36^{\prime} 00^{\prime \prime}$ & 880 & 1476 & $17,5^{\circ}$ & $\mathrm{Cfb}$ \\
\hline Itapeva, SP & 10 & $23^{\circ} 58^{\prime} 56^{\prime \prime}$ & $48^{\circ} 54^{\prime} 45^{\prime \prime}$ & 930 & 1254 & $18,9^{\circ}$ & Cwa \\
\hline Itararé, SP & 10 & $24^{\circ} 06^{\prime} 45^{\prime \prime}$ & $49^{\circ} 10^{\prime} 00^{\prime \prime}$ & 930 & 1306 & $19,0^{\circ}$ & Cfa \\
\hline Quatro Barras, PR & 10 & $25^{\circ} 21^{\prime} 56^{\prime \prime}$ & $49^{\circ} 04^{\prime} 37^{\prime \prime}$ & 915 & 1602 & $17,0^{\circ}$ & $\mathrm{Cfb}$ \\
\hline Três Barras, SC & 9 & $26^{\circ} 06^{\prime} 57^{\prime \prime}$ & $50^{\circ} 18^{\prime} 29^{\prime \prime}$ & 760 & 1440 & $17,1^{\circ}$ & $\mathrm{Cfb}$ \\
\hline
\end{tabular}

Lat. = latitude; Long. = longitude; Alt. = altitude; Prec. = precipitação média; Temp. Média = temperatura média. * Sementes coletadas em árvores que produziram tardiamente.

$$
\left.V O L=\left(\left(\pi * D A P^{2}\right)\right) / 40000\right) *(A L T * f f)
$$

em que o fator de forma ( ff ) utilizado foi de 0,6, segundo Sanquetta (2016). No ano de 2013, também foram realizadas avaliações dos caracteres de produção de pinhão, sendo estes: peso médio das pinhas ( $\mathrm{PMP}$, em kg), número de pinhões por pinha (NPP), peso unitário do pinhão ( $\mathrm{PP}, \mathrm{em} \mathrm{kg}$ ), peso médio dos pinhões por pinha (PMPP, em kg) e o peso das sementes vazias ( $\mathrm{PC}, \mathrm{em} \mathrm{kg}$ ).

\section{Estimativas dos parâmetros genéticos e análise de deviance}

As estimativas de componentes de variância e parâmetros genéticos dos caracteres quantitativos foram obtidas pelo método REML/BLUP (máxima verossimilhança restrita/melhor predição linear não viciada), aplicando-se o software genético-estatístico SELEGEN-REML/BLUP (RESENDE, 2007). Empregou-se o modelo 5 (modificado para uma planta por parcela), considerando o delineamento em blocos completos ao acaso, com várias procedências e progênies dentro de procedências, uma planta por parcela em um só local. Foram realizadas análises de deviance para determinar a variação entre procedências e progênies para todos os caracteres avaliados, conforme o modelo proposto por Resende (2007):

$$
y=\boldsymbol{X} r+\boldsymbol{Z} a+\boldsymbol{W} p+\boldsymbol{T}_{S}+e ;
$$


em que: y é o vetor de dados; $r$ o vetor dos efeitos de repetição (fixos) somados à média geral; $a$ o vetor dos efeitos genéticos aditivos individuais (progênies-aleatórios); $p$ o vetor dos efeitos de parcelas (fixos); s vetor dos efeitos de procedências (aleatórios); e o vetor de erros ou resíduos (aleatórios). As letras maiúsculas representam as matrizes de incidência para os referidos efeitos.

As estimativas de variâncias e parâmetros genéticos obtidas pelo programa foram:

a) Variância genética aditiva $\left(\hat{\sigma}_{a}^{2}\right) \hat{\sigma}_{a}^{2}=\left[\hat{a}^{\prime} A^{-1}+\hat{\sigma}_{e}^{2} \hat{a}^{\prime} \operatorname{tr}\left(A^{-1} \mathrm{C}^{22}\right)\right] / \mathrm{q}$

b) Variância residual $\left(\hat{\sigma}_{e}^{2}\right): \hat{\sigma}_{e}^{2}=\left[\mathrm{y}^{\prime} \mathrm{y}-\hat{b}^{\prime} \mathrm{X}^{\prime} \mathrm{y}-\hat{\mathrm{a}}^{\prime} \mathrm{Z}^{\prime} \mathrm{y}-\hat{r}^{\prime} \mathrm{Q}^{\prime} \mathrm{y}\right] /[\mathrm{N}-\mathrm{r}(\mathrm{x})]$

c) Herdabilidade individual no sentido restrito $\left(\hat{h}_{a}^{2}\right): \hat{h}_{a}^{2}=\frac{\hat{\sigma}_{a}^{2}}{\hat{\sigma}_{f}^{2}}$ d) Coeficiente de variação genética aditiva individual $\left(C V_{g i} \%\right): C V_{g i}(\%)=\frac{\sqrt{\hat{\sigma}_{a}^{2}}}{\hat{m}} \cdot 100$
$\hat{m}$ é a média geral do caráter.

e) Coeficiente de variação experimental $C V_{e}(\%): C V_{e}(\%)=\frac{\sqrt{\left[\left(0,75 \cdot \hat{\sigma}_{a}^{2}+\hat{\sigma}_{e}^{2}\right) / n\right]}}{\hat{m}} \cdot 100$

As correlações genotípicas e fenotípicas foram estimadas entre os caracteres quantitativos. Já entre os caracteres quantitativos e os de produção de pinhão foram obtidas somente as correlações fenotípicas. Esses parâmetros foram estimados usando os modelos 105 e 102 para correlação fenotípica e genética, respectivamente, conforme Resende, 2007 (Software Selegen). As significâncias das correlações foram verificadas pelo teste t utilizando o programa Genes (CRUZ, 2001).

Com base nos maiores valores genéticos preditos individuais (BLUP) foram propostas duas intensidades de seleção (24\% e 38\%), tendo por base o DAP aos 32 anos de idade (DAP 32), considerando a produção de madeira e a coleta de sementes para a próxima geração de melhoramento. Para tanto, foi considerado a proporção sexual dos indivíduos $(r=0,5)$ e as matrizes produtoras de sementes no ano de 2013.

O tamanho efetivo populacional $\left(N_{e}\right)$ foi estimado com base no método descrito por Vencovsky, Chaves, Crossa (2012) para espécies dioicas: $N_{e} \approx \frac{4 t}{D 4}$

em que: $t=f+m$ sendo $f$ e $m$ o número de fêmeas e número de machos selecionados, respectivamente;

$$
D_{4}=\frac{1}{r(1-r)}\left(\frac{N_{f}}{2 N_{f}-1}+\frac{N_{m}}{2 N_{m}-1}\right)+\frac{N_{f}}{2 N_{f}-1} \frac{2}{F}[(1-u) t-1]+\frac{N_{m}}{2 N_{m}-1} \frac{2}{M}[(1-v) t-1]
$$

sendo, $r$ = proporção sexual dos filhos; $N f$ e $N m$ : número de pais funcionais do sexo feminino e masculino da população de referência, respectivamente; $F$ e M: número total de pais do sexo feminino e masculino, respectivamente; $u$ e $v=$ fração de pais funcionais do sexo feminino e masculino, respectivamente, da população de referência, de tal modo que a fração, sendo: $u=\frac{F}{N f}$ em que: $(0<u \leq 1)$ e $v=\frac{M}{N m}$ em que: $(0<v \leq 1)$.

O ganho de seleção em porcentagem $\left(\hat{G}_{s} \%\right)$ com a seleção corresponde a:

$$
\hat{G}_{s}(\%)=\left(\frac{\sum_{\hat{a}}}{\sum k f} \times 100\right) \div \hat{m}
$$

em que a corresponde os valores genéticos preditos individuais (BLUP), $k f$ é o número médio de indivíduos selecionados por progênie e $\hat{m}$ é a média geral do caráter.

A diversidade genética após a seleção, foi quantificada conforme Wei e Lindgren (1996), citados por Resende (2002): $\left.D=\frac{N_{e f}}{N_{f o}}<D \leq 1\right)$; em que: $N_{f o}=$ número original de progênies; $N_{e f}=$ número efetivo de progênies selecionadas, sendo dado por: $N_{e f}=\frac{\left(\sum k_{f}\right)^{2}}{\sum k_{f}^{2}}$ em que: $k f=$ número médio de
indivíduos selecionados por progênie.

\section{RESULTADOS E DISCUSSÃO}

\section{Variação genética}

Diferenças significativas foram verificadas considerando os efeitos de procedências para ALT32, VOL32, DMC33 e ESP33 e os efeitos de progênies para o VOL32 (Tabela 2). Isto indica que o desbaste seletivo não restringiu a variação da população, e que existem procedências e progênies com crescimento superiores. Segundo Sebbenn et al. (2003) altos níveis de variação genética entre procedências são esperados, pois a ampla distribuição da espécie, associada ao isolamento reprodutivo de populações 
localizadas a grandes distâncias, provavelmenteé a principal causa da elevada diferenciação. Diferenças significativas para os efeitos de procedências, sugerem a existência de diferenças geneticamente fixadas visto que, para a instalação do teste foram utilizadas sementes de 12 origens, abrangendo grande parte da área de ocorrência natural da espécie. As diferenças entre latitudes, clima, índices pluviométricos entre as localidades de coletas de sementes podem originar à partir das forças evolucionárias indivíduos com características de adaptação local. Diferenças genéticas em nível de procedências e progênies tem sido reportada por Duarte et al. (2012), Sebbenn, Freitas e Freitas[REMOVED HYPERLINK FIELD] (2004), Shimizu (1999), Sousa, Robinson e Hattemer (2004) e Stefenon; Finkeldey (2007). Tais diferenças permitem a obtenção de progressos genéticos com a seleção de indivíduos tanto das procedências quanto das progênies mais adaptadas e produtivas para o local de estudo.

Tabela 2. Estimativas de parâmetros genéticos para os caracteres silviculturais aos 30, 32 e 33 anos.

Table 2. Estimates of genetic parameters for silvicultural traits at age 30, 32 and 33 years.

\begin{tabular}{lccccc}
\hline \multicolumn{1}{c}{ Caracteres } & $\begin{array}{c}\text { LRT }\left(\mathbf{X}^{2}\right) \\
\text { Proc }\end{array}$ & $\begin{array}{c}\text { LRT }\left(\mathbf{X}^{2}\right) \\
\text { Prog }\end{array}$ & $\boldsymbol{C} \boldsymbol{V}_{g i}(\%)$ & $\boldsymbol{C V}(\%)$ & $\hat{\boldsymbol{h}}_{\boldsymbol{a}}^{2}$ \\
\hline DAP30 $(\mathbf{c m})$ & 1,44 & 2,61 & 10,4 & 5,1 & $0,59 \pm 0,30$ \\
DAP32 $(\mathbf{c m})$ & 1,28 & 2,59 & 10,2 & 5,1 & $0,58 \pm 0,29$ \\
ALT32 $(\mathbf{m})$ & $13,46^{* *}$ & 0,00 & 1,0 & 3,7 & $0,10 \pm 0,04$ \\
VOL32 $\left(\mathbf{m}^{3} \cdot\right.$ arv $\left.^{-1}\right)$ & $2,82^{*}$ & $2,77^{*}$ & 24,0 & 11,5 & $0,60 \pm 0,30$ \\
DMC33 $(\mathbf{m})$ & $3,69^{*}$ & 0,00 & 2,9 & 6,8 & $0,03 \pm 0,06$ \\
ESP33 $(\mathbf{c m})$ & $3,09^{*}$ & 0,04 & 5,9 & 8,9 & $0,07 \pm 0,10$ \\
\hline
\end{tabular}

LRT: teste da razão de verossimilhança; $X^{2}$ qui quadrado da deviance.* LRT $<0,05$; ** LRT $<0,0$, com 0,5 grau de liberdade; DAP30 e DAP32 = diâmetro a altura do peito avaliado aos 30 e 32 anos, respectivamente; ALT32= altura avaliada 32 anos; VOL32= volume cilíndrico avaliado aos 32 anos; DMC33 = diâmetro médio da copa avaliado aos 33 anos; ESP33 = espessura da casca avaliada aos 33 anos de idade; $C V_{\text {gi }}$ e $C V_{e}$ são os coeficientes de variação genética aditiva individual e de variação residual, respectivamente; $\hat{h}_{a}^{2}$ é a herdabilidade individual no sentido

\section{Taxa de crescimento}

As médias gerais para os caracteres de crescimento foram de $38,03 \mathrm{~cm}, 40,83 \mathrm{~cm}, 23,21 \mathrm{~m}$, 1,87 $\mathrm{m}^{3} \cdot \operatorname{arv}^{-1}, 7,09 \mathrm{~m}$ e 2,73 para DAP30, DAP32, ALT32, VOL32, DMC33 e ESP33 (Tabela 3). Para a mesma espécie, aos 30 anos de idade, Sebbenn, Freitas e Freitas (2004) obtiveram os valores de 20,47 cm; 18,12 m e 0,38 $\mathrm{m}^{3}$. arv $^{-1}$ para DAP, ALT e VOL, respectivamente, em Itapeva-SP. Desempenho inferior ao do presente estudo, foi observado por Hentz et al. (2016) em um plantio de araucária de aproximadamente 60 anos, nas condições edafoclimáticas de Santa-Maria-RS, para DAP $(33,27 \mathrm{~cm})$ e ALT (19,67 m). Segundo Duarte et al. (2012), o crescimento das espécies arbóreas está diretamente relacionado a localização dos sítios em estudo e as condições específicas para o desenvolvimento encontrado em cada um deles, assim como o manejo florestal adotado. Diante disso, com base nos resultados do estudo, verificou-se um bom desempenho em crescimento para a espécie nas condições de Colombo-PR.

Tabela 3. Médias para diâmetro a altura do peito (DAP, em cm), altura ( $A L T$, em m), volume (em $\mathrm{m}^{3}$.arv ${ }^{-1}$ ), diâmetro médio de copa (DMC, em $\mathrm{m}$ ) e espessura da casca (ESP, em $\mathrm{cm})$, mensurados aos 30, 32 e 33 anos, em um teste de procedências e progênies de $A$. angustifolia, em Colombo-PR.

Table 3. Average of diameter at breast height $\left(\mathrm{DBH}\right.$, in $\mathrm{cm}$ ), height ( $A L T$, in $\mathrm{m}$ ), volume (in $\mathrm{m}^{3} . \mathrm{arv}^{-1}$ ), mean crown diameter (DMC, in $\mathrm{m}$ ) and bark thickness (ESP, in $\mathrm{cm}$ ), measured at age 30, 32 and 33 years, in provenances and progenies test of $A$. angustifolia at Colombo municipality, Paraná State.

\begin{tabular}{|c|c|c|c|c|c|c|}
\hline & \multirow{2}{*}{$\begin{array}{c}30 \text { anos } \\
\text { DAP }\end{array}$} & \multicolumn{3}{|c|}{32 anos } & \multicolumn{2}{|c|}{33 anos } \\
\hline & & DAP & ALT & VOL & DMC & ESP \\
\hline Média & 38,03 & 40,83 & 23,21 & 1,87 & 7,09 & 2,73 \\
\hline IMA & {$[1,26]$} & {$[1,27]$} & {$[0,72]$} & - & - & - \\
\hline
\end{tabular}

[IMA]: incremento médio anual; [DAP]: cm/ano; [ALT]: m/ano.

Os incrementos médios anuais (IMA) para DAP30 (1,26 cm), DAP32 $(1,27 \mathrm{~cm})$ e ALT32 $(0,72 \mathrm{~m})$ foram inferiores ao valor sugerido para a espécie em sítios adequados $(1 \mathrm{~m}$ em altura e 1,5 $\mathrm{cm}$ a $2,0 \mathrm{~cm}$ em DAP) (CARVALHO, 1994). Baixos valores de IMA para a espécie também foram observados por Hess, Schneider e Finger, (2009) e Sebbenn, Freitas e Freitas (2004). Por outro lado, resultados 
Silva et al. - Seleção de procedências e progênies de Araucaria angustifolia

(Bert.) O. Kuntze para produção de madeira e pinhão

superiores aos obtidos no presente estudo foram observados por Kageyama e Jacob (1980) para DAP $(1,7-1,9 \mathrm{~cm})$ aos 3,5 anos e por Sebbenn, Freitas e Freitas (2004) para ALT (1,99 m) aos 6 anos, ambos para testes de A. angustifolia. Valores superiores foram obtidos para o incremento médio anual em espécies exóticas, principalmente as dos gêneros Pinus e Eucalyptus, em idades precoces (MACHADO et al., 2010; ROSADO et al., 2012; TOMAZELLO FILHO et al., 2017). Comparando o IMA da A. angustifolia com outras espécies florestais nativas, o crescimento foi superior a Balfourodendron riedelianum (pau marfim) para DAP (SEBBENN et al., 2007), Cariniana legalis (jequitibá-rosa) para ALT e DAP (SEBBENN et al., 2009) e Myracrodruon urundeuva (aroeira) para DAP (PUPIN et al., 2017). O crescimento lento de algumas espécies arbóreas, embora seja uma característica desfavorável à silvicultura, por gerar menor receita anual, é uma característica favorável à formação de madeira de alta qualidade (SEBBENN et al., 2009).

\section{Parâmetros genéticos}

O coeficiente de variação experimental $\left(C V_{e}\right)$ foi considerado de baixa magnitude $(\leq 11,5 \%)$, evidenciando condições experimentais adequadas de controle ambiental (Tabela 2). Valores de coeficiente de variação experimental na ordem de 10 a 20\% podem ser considerados baixos para experimentos de campo (PIMENTEL-GOMES; GARCIA, 2002). O coeficiente de variação genética individual $\left(\mathrm{CV}_{g i}\right)$ foi alto para DAP30, DAP32 e VOL32 $(\geq 10,2 \%)$ (Tabela 2). Os coeficientes de variação genética acima de 7\% são considerados altos (SEBBENN et al., 1998).

Os caracteres VOL32, DAP30 e DAP32 foram os que apresentaram maior variabilidade genética entre progênies. Portanto, durante o processo de seleção há possibilidade de obter-se maiores ganhos genéticos considerando estes caracteres. Os coeficientes de herdabilidade individual no sentido restrito $\left(\hat{h}_{a}^{2}\right)$ foram variáveis para os caracteres apresentando herdabilidades de baixa a alta magnitude, variando de 0,03 (DMC33) a 0,59 (DAP30). Valores de herdabilidades altos a medianos indicam que o controle genético para os caracteres é expressivo, e há possibilidade de ganhos genéticos com a seleção. Sebbenn et al. (2003) observaram coeficientes de herdabilidade individual baixos para os caracteres DAP $(0,059), \operatorname{ALT}(0,053)$ e VOL $(0,053)$ ao avaliarem um teste de procedências e progênies de A. angustifolia em Itapeva-SP. É importante ressaltar que a herdabilidade é uma propriedade não somente de um caráter, mas também da população e circunstâncias de ambiente às quais os indivíduos estão sujeitos. Uma vez que a herdabilidade depende da magnitude de todos os componentes de variância uma alteração em qualquer um deles afetará o seu valor (FALCONER, 1987). As acurácias obtidas foram consideradas de moderada a alta magnitude $(\geq 0,57)$ indicando alta precisão no acesso a variação genética verdadeira a partir da variação fenotípica observada, principalmente, para os caracteres relacionados ao volume de madeira.

\section{Análise dos caracteres de produção de pinhão}

As médias para os caracteres de produção de pinhão avaliados em 19 matrizes produtoras no ano de 2013 foram de 1,40 kg para peso médio das pinhas (PMP), 88,78 pinhões por pinha (NPP), 0,006 kg para peso unitário dos pinhões (PP), 0,59 kg para peso médio de pinhões por pinhas (PMPP) e 0,74 kg para o peso dos pinhões vazios (PC) (Tabela 4). Valores inferiores foram obtidos por Figueiredo Filho et al. (2011) em uma área plantada para PMP (1,39 kg); NPP (57,47), PP (5,80 g) e PMPP $(0,37 \mathrm{~kg})$. Mantovani, Morellato e Reis (2004) também obtiveram baixa produção de pinhão em dois anos consecutivos (2001 e 2002) em uma população natural de araucária (93,9 e 80,7 pinhões (NPP), 6,58 g e 7,00 g (PP). A produção de pinhão é variável tanto em áreas plantadas e naturais podendo ser afetada por inúmeros fatores. Segundo Figueiredo Filho et al. (2011), em condições naturais, fatores como o número de indivíduos, proporção sexual e grau de evolução da floresta são determinantes na produção de pinhões, bem como a idade das árvores, por definir o período, intensidade e a frutificação (SOUSA; HATTEMER, 2003). Segundo Krupt et al. (2010), a variação na quantidade de sementes produzidas em cada um dos estróbilos está relacionada aos eventos de polinização, mas particularmente às dificuldades enfrentadas durante o processo. Os grãos de pólen de A. angustifolia, transportados pelo vento, são relativamente grandes $(61,50 \mathrm{~mm})$, apresentando uma redução na capacidade de flotação (SOUSA, 2001). Para a espécie, os estudos realizados visando determinar a distância média de dispersão do pólen mostraram os valores médios de até 200 m (BITTENCOURT; SEBBENN, 2008) com uma taxa de polinização de 54\%; 124 m (SANT' ANA et al., 2013). Bittencourt e Sebbenn (2007) observaram que em populações separadas os grãos de pólen podem ser dispersos até distâncias de $2 \mathrm{~km}$. 
Tabela 4. Estatística descritiva dos caracteres de produção de pinhão no teste de $A$. angustifolia em Colombo-PR, no ano de 2013.

Table 4. Descriptive statistics of seed production at age 33 years in provenances and progenies test of $A$. angustifolia at Colombo municipality, Paraná State.

\begin{tabular}{ccccc}
\hline Caracteres & Média & $\begin{array}{c}\text { Desvio } \\
\text { Padrão }\end{array}$ & Mínimo & Máximo \\
\hline PMP (kg) & 1,40 & 0,32 & 0,91 & 2,08 \\
NPP & 88,78 & 28,03 & 20,92 & 132,17 \\
PP (Kg) & 0,006 & 0,001 & 0,005 & 0,010 \\
PMPP (kg) & 0,592 & 0,21 & 0,11 & 1,03 \\
PC (Kg) & 0,74 & 0,144 & 0,56 & 1,08 \\
\hline
\end{tabular}

PMP $(\mathrm{kg})=$ Peso médio das pinhas; NPP = Número de pinhões por pinha; PP $(\mathrm{kg})=$ Peso unitário do pinhão; PMPP $(\mathrm{kg})=$ Peso médio de pinhões por pinha $(\mathrm{kg})$ : $\mathrm{PC}(\mathrm{kg})=$ peso dos pinhões vazios.

De modo geral, os caracteres de produção de pinhão apresentam diferenças fenotípicas expressivas, o que pode contribuir para resultados significativos nos próximos ciclos de melhoramento. O baixo número de matrizes produtoras no ano de 2013 (19 matrizes), pode estar relacionada principalmente pela assincronia entre a liberação de pólen e a maturação das árvores femininas, uma vez que o teste é composto por progênies de várias localidades, apresentando assim, progênies com a formação de estruturas reprodutivas mais precoce do que outras, comprometendo o fluxo de pólen entre os indivíduos.

O peso dos pinhões vazios (PC) representou 44\% do peso médio da pinha. Valores inferiores foram encontrados por Mantovani, Morellato e Reis (2004) em plantios florestais $(22,6 \%)$ e por Figueiredo Filho et al. (2011) em áreas naturais $(28,8 \%)$ e em áreas plantadas $(22,6 \%)$. A alta proporção de pinhões vazios pode estar associada a fatores como: baixa produção de pólen dentro da área observada, a nuvem de pólen produzida pelas árvores pode, ainda, não ter sido suficientemente grande para permitir uma distribuição homogênea, a alta taxa de aborto de óvulos ou embriões em árvores jovens (SOUSA; HATTEMER, 2003). Devido ao ciclo da produção da araucária ocorrer com anos de contra safra após dois ou três anos consecutivos de alta produção de sementes (CARVALHO, 2002), para a obtenção de resultados mais fidedignos, recomenda-se que a produção de sementes da espécie seja acompanhada por no mínimo três anos consecutivos.

\section{Correlação fenotípica e genética entre caracteres de crescimento}

As correlações fenotípicas e genéticas entre os caracteres de crescimento foram positivas e significativas, e consideradas moderadas a altas (Figura 1). A correlação fenotípica e genética entre o DAP32 vs VOL32 foram as mais altas (0,97 e 0,96, respectivamente). Correlações de alta magnitude entre um par de caracteres é de grande interesse, uma vez, que os mesmos podem ser considerados como um único caráter na seleção, sem grandes prejuízos para qualquer um desses (VENCOVSKY, 1978). Os resultados sugerem que a seleção considerando tanto o DAP32 quanto o VOL32 pode promover ganhos em um deles por meio da seleção indireta do outro. Correlações genéticas altas e positivas foram também observadas para a espécie em testes de procedências e progênies entre DAP vs ALT, DAP vs VOL, VOL vs ALT avaliada aos 9, 11 e 23 anos (SHIMIZU, 1999) e aos 21 anos (SEBBENN et al., 2003). De maneira geral, a correlação genética entre os caracteres de crescimento é alta em espécies florestais. Valores próximos a um sugere que há um elevado grau de pleiotropia entre os caracteres. Isso significa que muitos locos gênicos comuns afetam ambos os caracteres (WHITE et al., 2007).

Observou-se ainda correlação positiva e significativa entre DAP32 vs ESP33 $(0,51)$. Porém, essa correlação não é desejável uma vez que quanto maior a espessura da casca maior a quantidade de resíduos que pode gerar durante o processamento mecânico, acarretando menores rendimentos. No melhoramento genético da A. angustifolia, o caráter espessura de casca deve ser priorizado, principalmente para produção de madeira para desdobro, pois o rendimento em volume de madeira é afetado negativamente. 


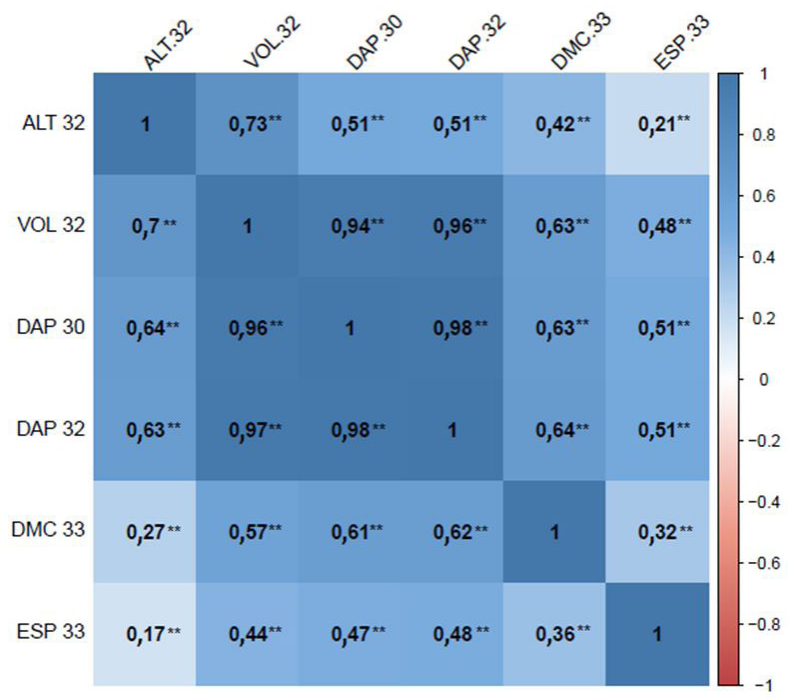

** Significativo a $1 \%$ de probabilidade pelo Teste $t$. DAP 30; DAP 32: diâmetro a altura do peito avaliado aos 30 e 32 anos; ALT 32: altura avaliada 32 anos; VOL 32: volume total avaliado aos 32 anos; DMC 33: diâmetro médio da copa avaliado aos 33 anos; ESP 33: espessura da casca avaliada aos 33 anos de idade.

Figura 1. Estimativas das correlações genéticas (acima da diagonal) e fenotípicas (abaixo da diagonal) entre os caracteres de crescimento em progênies de $A$. angustifolia avaliados aos 30, 32 e 33 anos de idade em Colombo-PR.

Figure 1. Estimates of genetic (above diagonal) and phenotypic (below diagonal) correlations between growth traits evaluated at age 30,32 and 33 years in provenances and progenies test of Araucaria angustifolia at Colombo municipality, Paraná State.

\section{Correlação fenotípica entre caracteres de crescimento e produção de pinhão}

As correlações fenotípicas entre os caracteres de crescimento e os relacionados a produção de pinhão foram significativas (Figura 2), variando de -0,35 (ESP33 vs PMP) a 0,34 (ESP33 vs PC). Se um caráter estiver correlacionado negativamente com alguns e positivamente com outros caracteres deve-se evitar a seleção desse caráter, visto que essa seleção pode levar a resultados indesejáveis na população de melhoramento em gerações subsequentes (FALCONER; MACKAY, 1996).

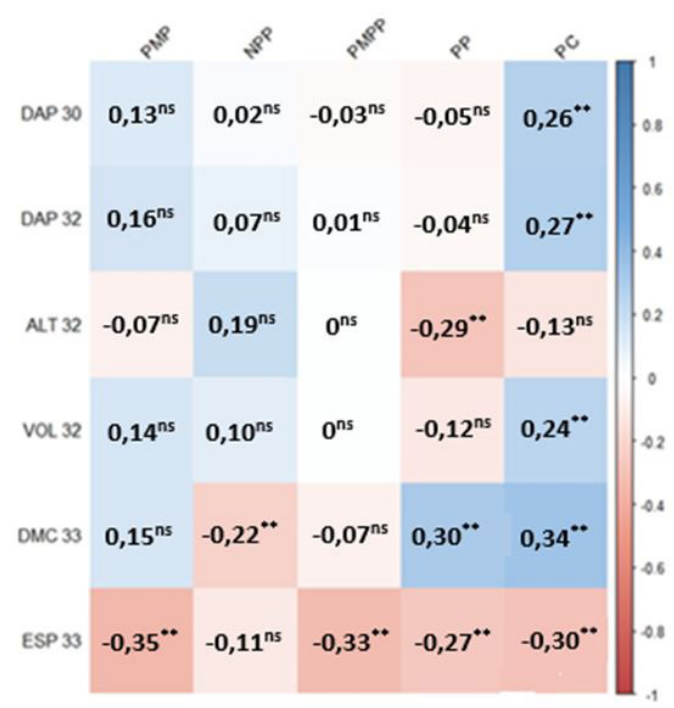

** Significativo a $1 \%$ de probabilidade pelo Teste $t$. ${ }^{n s}$ não significativo. PMP $(\mathrm{kg})=$ Peso médio das pinhas; NPP= Número de pinhões por pinha; PP $(\mathrm{kg})=$ Peso unitário do pinhão; PMPP $(\mathrm{kg})=$ Peso médio de pinhões por pinha $(\mathrm{kg})$ : PC $(\mathrm{kg})=$ peso dos pinhões vazios; DAP30 $(\mathrm{cm})$ e DAP32 $(\mathrm{cm})=$ diâmetro a altura do peito aos 30 e 32 anos, respectivamente; ALT32 (m)= altura total aos 32 anos; VOL32 $\left(\mathrm{m}^{3} \cdot \operatorname{arv}^{-1}\right)=$ volume total; DMC33 $(\mathrm{m})=$ diâmetro médio da copa aos 33 anos; ESP33 (cm)= espessura da casca aos 33 anos de idade.

Figura 2. Estimativas das correlações fenotípicas entres os caracteres de crescimento e de produção de pinhão no teste de $A$. angustifolia em Colombo PR.

Figure 2. Estimates of the phenotypic correlations between growth and seed production traits in provenances and progenies test of Araucaria angustifolia at Colombo municipality, Paraná State. 
Observou-se relação significativa entre o DMC33 e os caracteres PMP $(0,15)$ e PP $(0,30)$. Assim, quanto maior o diâmetro médio de copa da árvore, maior será o peso das pinhas e o peso unitário dos pinhões e, consequentemente menor será o número de pinhões por pinha. No geral, as melhores matrizes produtoras são as árvores dominantes, com copas vigorosas e amplas e expostas a luz, devido a maior disponibilidade de recursos, resultado de espaçamentos menos adensados, livres de competição com outras árvores. De acordo com os resultados de correlação entre os caracteres quantitativos e de produção de pinhão, sugere-se que para a implantação de um pomar de sementes ou até mesmo de plantios comerciais, os dois objetivos podem ser considerados conjuntamente na primeira geração, porém após avaliações, as estratégias deverão seguir separadamente até obtenção dos genótipos melhorados. Recomenda-se o acompanhamento e avaliações em anos posteriores para a obtenção de resultados mais fidedignos considerando as diferentes safras de produção.

\section{Ganho de seleção}

Os valores de Gs (\%) foram de 9,5\% e 7,6\% para a intensidade de seleção de 24 e 38\%, respectivamente (Tabela 5). Com a menor intensidade de seleção, observou-se menor ganho genético, porém parte considerável da diversidade genética $(0,49)$ e o tamanho efetivo populacional de 55 foram mantidos. Comparando as duas intensidades de seleção a queda do ganho genético $(1,9 \%)$ foi relativamente pequena quando se mantém maior parte da diversidade genética. Assim, para a seleção visando à coleta de sementes para as próximas gerações de melhoramento, sugere-se adotar intensidades menores que não comprometa a diversidade genética, já que o tamanho efetivo populacional se encontra no limite daquele que se tem recomendado para cada geração de melhoramento $(\mathrm{Ne}=50)$. Esse valor é recomendado para evitar a depressão endogâmica (FRANKEL; SOULÉ, 1981), bem como reduzir a perda de alelos favoráveis ao longo dos ciclos de seleção (RESENDE, 1999). A existência de árvores de ambos os sexos próximas entre si, aumenta a probabilidade de ocorrer maior número de polinizações, e consequentemente, o sucesso reprodutivo da espécie. Outro fator importante a ser considerado na seleção é a proporção sexual com adequada disposição espacial das árvores para facilitar a dispersão da nuvem de pólen e, consequentemente o cruzamento entre os indivíduos do pomar de sementes.

Tabela 5. Progresso esperado mediante ao ganho de seleção $\left(\hat{G}_{s} \%\right)$, tamanho efetivo populacional $\left(N_{e}\right)$ e a diversidade genética $(D)$, considerando a razão sexual de descendentes de 0,5 em teste de procedências e progênies de $A$. angustifolia em Colombo-PR.

Table 5. Progress expected through gain by selection $\left(\hat{G}_{s} \%\right)$, effective population size $(\mathrm{Ne})$ and genetic diversity $(D)$, considering the sexual ratio of offspring of 0.5 in provenances and progenies test of $A$. angustifolia at Colombo municipality, Paraná State.

\begin{tabular}{ccccccc}
\hline $\boldsymbol{f}+\boldsymbol{m}$ & $\boldsymbol{N}_{\boldsymbol{e}}$ & $\hat{\mathrm{a}}(\mathbf{c m})$ & $\begin{array}{c}\boldsymbol{\mu} \\
(\mathbf{c m})\end{array}$ & $\hat{\boldsymbol{G}}_{\boldsymbol{s}}(\boldsymbol{\%})$ & $\boldsymbol{D}$ & $\begin{array}{c}\text { Intensidade de } \\
\text { seleção (\%) }\end{array}$ \\
\hline 50 & 38,78 & 4,48 & 47,31 & 9,5 & 0,31 & 24 \\
80 & 54,57 & 3,50 & 45,94 & 7,6 & 0,49 & 38 \\
\hline
\end{tabular}

$F$ e $M=(77)=$ número de indivíduos femininos e masculinos potencialmente funcionais nas populações, respectivamente; $u$ e $v=(0,09)=$ é a fração de genitores femininos e masculinos funcionais em relação ao número de indivíduos também femininos e masculinos nas populações de referência, respectivamente, sendo $0<u \leq \mathrm{I}$ e $0<v \leq \mathrm{I} ; f+m=$ número de indivíduos femininos e masculinos descendentes; $\mu$ : média geral fenotípica ; â: efeito genético aditivo.

Ainda com base no valor genotípico do caráter DAP32 foi realizada o ranqueamento das melhores procedências (Tabela 6). Os maiores ganhos genotípicos preditos (Tabela 6) foram observados para as procedências de Irati-PR $(42,3 \mathrm{~cm})$ e Itapeva-SP $(41,4 \mathrm{~cm})$. Esses resultados demonstram o forte efeito das procedências em relação ao crescimento das árvores. A escolha da procedência pode ter grande influência no aumento da produtividade no presente local de experimentação. Para os próximos ciclos de melhoramento de araucária para produção de madeira, os indivíduos mais produtivos dessas procedências deverão ser priorizados, tanto para esse fim quanto para a conservação, nas condições ambientais de Colombo-PR.

No geral as procedências de menores altitudes e de maior temperatura média anual apresentaram maior crescimento em DAP32 na região do estudo. A procedência Campos do Jordão-SP apresentou o menor valor genotípico para o caráter DAP32 (39,64). Dentre os fatores citados por Martinkoski et al. (2015), as principais variáveis climáticas que apresentam maior influência sobre o crescimento da espécie 
Silva et al. - Seleção de procedências e progênies de Araucaria angustifolia

(Bert.) O. Kuntze para produção de madeira e pinhão

Tabela 6. Seleção das melhores procedências no teste de $A$. angustifolia em Colombo-PR, considerando o DAP aos 32 anos de idade.

Table 6. Selection of the best provenances in provenances and progenies test of Araucaria angustifolia at Colombo municipality, Paraná State., considering DAP at age 32 years.

\begin{tabular}{clccc}
\hline Ordem & \multicolumn{1}{c}{ Procedências } & $\boldsymbol{u}+\boldsymbol{g}$ & Ganho & Nova média \\
\hline 1 & Irati, PR (tardio) & 42,2929 & 1,4622 & 42,2929 \\
2 & Itapeva, SP & 41,3955 & 1,0135 & 41,8442 \\
3 & Itararé, SP & 41,1670 & 0,7878 & 41,6185 \\
4 & Chapecó, SC & 40,8543 & 0,5967 & 41,4275 \\
5 & Três Barras, SC & 40,8313 & 0,4775 & 41,3082 \\
6 & Irati, PR & 40,8282 & 0,3975 & 41,2282 \\
7 & Congonhal, MG & 40,7114 & 0,3237 & 41,1544 \\
8 & Quatro Barras, PR & 40,6686 & 0,2629 & 41,0937 \\
9 & Barbacena, MG & 40,5789 & 0,2057 & 41,0365 \\
10 & Ipuiúna de Caldas, MG & 40,5148 & 0,1536 & 40,9843 \\
11 & Caçador, SC & 40,4789 & 0,1076 & 40,9384 \\
12 & Campos do Jordão, SP & 39,6468 & 0,0000 & 40,8307 \\
\hline
\end{tabular}

$u+g=$ média genotípica ou valores genotípicos; Ganho (\%); Nova média $(\mathrm{cm})$.

são a precipitação e as temperaturas médias. Diante dessas informações, é possível observar que a precipitação média anual inferior no local do teste, se comparado com o local da procedência, pode ter contribuído para expressar menor crescimento dessa procedência em Colombo-PR. Zanon e Finger (2010), observaram que a precipitação é um fator que influiu positivamente no incremento médio anual da espécie, pois o crescimento decresceu nos meses de março e estagnando nos meses de julho a agosto, em que a quantidade de chuva foi abaixo da média. Estas respostas ocorrem, devido a menor pluviosidade, restringindo os processos fisiológicos.

Para a formação de uma população de melhoramento, recomenda-se a mistura de sementes de araucária de várias procedências, garantindo a variação genética, estimulando o cruzamento entre indivíduos não aparentados, afim de promover recombinações entre possíveis raças geográficas ou indivíduos com adaptação local.

\section{CONCLUSÕES}

A existência de variação genética em nível de procedências para os caracteres quantitativos indica a possibilidade de ganhos genéticos satisfatórios por meio da seleção.

A correlação fenotípica e genética foi alta entre os caracteres DAP32 vs VOL32, dessa forma, ambos os caracteres podem ser considerados para a seleção, promovendo ganhos com a seleção indireta do outro.

A correlação fenotípica entre os caracteres de crescimento e os relacionados a produção de pinhão foi significativa para poucas associações, sugere-se que a produção de madeira e pinhão no processo de seleção genética, sejam considerados conjuntamente apenas na primeira geração.

A seleção de individual de 38\%, para a produção de madeira e sementes, é a mais indicada pois pode gerar ganhos genéticos e manter níveis adequados do tamanho efetivo populacional.

As procedências de Irati-PR e Itapeva-SP apresentam melhor desempenho em crescimento e valor genotípico, assim, são as mais indicadas para plantios em áreas da região de Colombo-PR.

\section{REFERÊNCIAS}

ANSELMINI, J. I.; ZANETTE, F. Polinização controlada em Araucaria angustifolia. Cerne, Lavras, v. 18, n. 2, p. 247-255, 2012.

BITTENCOURT, J. V. M.; SEBBENN, A. M. Pollen movement and spatial genetic structure in a continuous forest of wind-pollinated Araucaria angustifolia, inferred from paternity and TWOGENER analysis. Conservation Genetics, v. 9, p. 855-868, 2008.

BITTENCOURT, J. V. M.; SEBBENN, A. M . Patterns of pollen and seed dispersal in a small, fragmented population of the wind-pollinated tree Araucaria angustifolia in southern Brazil. Heredity, v. 99, p. 580-591, 2007. 
CARVALHO, P. E. R. Espécies florestais brasileiras: recomendações silviculturais, potencial idades e uso de madeira. Colombo: Embrapa lCNPF; Brasília, 1994. 640 p.

CARVAlHO, P. E. R. Pinheiro-do-paraná. Colombo: Embrapa Florestas, 2002. p. 17. (Circular Técnica, 60).

CRUZ, C. D. Programa GENES - versão windows. Aplicativo computacional em Genética e Estatística. Viçosa: Editora UFV, 2001. v. 1. 648 p.

DUARTE, R. I.; SILVA, F. A. L. S.; SCHULTZ, J.; SILVA, J, Z.; REIS, M. S. Características de Desenvolvimento Inicial em Teste de Progênie de uma População de Araucária na Flona de Três Barras-SC. Biodiversidade Brasileira, Brasília, v. 2, n. 2, p. 114-123, 2012.

EMBRAPA - EMPRESA BRASILEIRA DE PESQUISA AGROPECUÁRIA. Sistema brasileiro de classificação de solos. 3. ed. Brasília: Embrapa Produção de Informação, 2013. p. 353.

FALCONER, D. S. Introdução à genética quantitativa. Viçosa: UFV, 1987. p. 219.

FALCONER, D. S.; MACKAY, T. F. C. Introduction to quantitative genetics. London: Longman Malaysia, 1996. p. 46.

FIGUEIREDO-FILHO, A.; ORELLANA, E.; NASCIMENTO, F.; DIAS, A. N.; INOUE, M. T. Produção de sementes de Araucaria angustifolia em plantio e em floresta natural no centro-sul do Estado do Paraná. Revista Floresta, Curitiba, v. 41, n. 1, p. 153-160, 2011.

FONTES, B. P. D.; DAVIDE, L. C.; DAVIDE, A. C. Fisiologia e citogenética de sementes envelhecidas de Araucaria angustifolia. Ciência e Agrotecnologia, Lavras, v. 25, n. 2, p. 346-355, 2001.

FRANKEL, O. H.; SOULÉ, M. S. Conservation and evolution. Cambridge: Cambridge University Press, 1981. p.327.

GARTLAND, K. M. A.; CROW, R. M.; FENNING, T. M.; GARTLAND, J. S. Genetically modified trees: production, properties, and potential. Journal of Arboriculture, Champaign, v. 29, n. 5, p. 259-266, 2003.

GUERRA, M. P.; SILVEIRA, V.; REIS, M. S.; SCHENEIDER, L. Exploração, manejo e conservação da araucária (Araucaria angustifolia). In: SIMÕES, L. L.; LINO, C. F. (Orgs.). Sustentável Mata Atlântica: a exploração dos seus recursos florestais. São Paulo: SENAC, 2002. p. 85-102.

HENTZ, A. M. K.; PASA, D. L.; TALGATTI, M.; FERREIRA A. R. C.; MELO FILHO, J. A. Distribuição diamétrica e determinação da altura em plantio de Araucaria angustifolia (bertol.) kuntze na região central do Rio Grande do Sul. Scientia Plena, Aracajú, v. 12, n. 1, 2016.

HESS, A. F.; SCHNEIDER, P. R.; FINGER, C. A. G. Crescimento em diâmetro de Araucaria angustifolia (Bertol.) Kuntze em função da idade, em três regiões do Rio Grande do Sul. Ciência Florestal, Santa Maria, v. 19, n. 1, p. 7-22, 2009.

HESS, A. F.; CALGAROTTO, A. R.; PINHEIRO, R.; WANGINIAK, T. C. R. Proposta de manejo de Araucaria angustifolia utilizando o quociente de Liocourt e análise de incremento, em propriedade rural no Município de Lages, Santa Catarina. Pesquisa Florestal Brasileira, Colombo, v. 30, n. 64, p. 337-345, 2010.

IBÁ. Relatório IBÁ 2015. Brasília: IBÁ, 2015. p. 65.

KAGEYAMA, P. Y.; JACOB, W. S. Variação genética entre e dentro de populações de Araucaria angustifolia (Bert) O. Ktze. In: ENCONTRO IUFRO SOBRE A PROBLEMATICA DA ARAUCÁRIA, 1., 1980, Curitiba. Anais... Curitiba: FUPEF, 1980. n. 115, p. 83-86.

KRUPEK, R. A.; Ribeiro, V. Biometria e Germinação de Sementes de Araucaria angustifolia (Bert.) O. Kuntze Provenientes de um Remanescente Florestal do Município de Turvo (PR). Revista Ciências Exatas e Naturais, v.12, n. 1, p. 73- 89, 2010.

KUHN, S. A.; ERNESTO, J.; MARIATH, J. E. A. Reproductive biology of the Brazilian pine (Araucaria angustifolia - Araucariaceae): development of microspores and microgametophytes. Flora, Amsterdam, v. 209, n. 5-6, p. 290-298, 2014.

Sci. For., Piracicaba, v. 46, n. 120, p. 519-531, dez. 2018 DOI: dx.doi.org/10.18671/scifor.v46n120.01 
Silva et al. - Seleção de procedências e progênies de Araucaria angustifolia

(Bert.) O. Kuntze para produção de madeira e pinhão

MACHADO S. A., FIGURA MA, SILVA L. C. R, NASCIMENTO R. G. M., QUIRINO S. M. S., TÉO S. J. Dinâmica de crescimento de plantios jovens de Araucaria angustifolia e Pinus taeda. Pesquisa Florestal Brasileira, v. 30, n.62, p. 165-170, 2010.

MANTOVANI, A.; MORELLATO, L. P. C.; REIS, M. S. Fenologia reprodutiva e produção de sementes em Araucaria angustifolia (Bert.) O. Kuntze. Revista Brasileira de Botânica, São Paulo, v. 27, n.4, p. 787-796, 2004.

MARTINKOSKI, L.; VOGEL, G. F.; JADOSKI, SIDNEI OSMAR. Influência do clima no crescimento diamétrico de Araucaria angustifolia: revisão bibliográfica. Pesquisa Aplicada e Agrotecnologia (Online), v. 8, p. 104111, 2015.

MARTINS, A. P. M.; MACHADO, S. A.; FIGUEIREDO FILHO, A.; DALLA CORTE, A. P.; GORENSTEIN, M. R. Efeito da idade na forma do fuste de Araucaria angustifolia na região Centro-Sul do Paraná. Pesquisa Florestal Brasileira, Brasília, v. 37, p. 109-117, 2017.

LORENZI, H. Árvores brasileiras: manual de identificação e cultivo de plantas nativas do Brasil. 3 ed. Nova Odessa: Plantarum, 2000.

PIMENTEL-GOMES, F.; GARCIA, C. H. Estatística aplicada a experimentos agronômicos e florestais: exposição com exemplos e orientações para uso de aplicativos. Piracicaba: FEALQ, 2002, 309 p.

PUPIN, S.; FREITAS, M.L.M.; CANUTO, D.S.O.; SILVA, A. M.; ALZATE-MARIN, A. L. MORAES, M. L. T. Variabilidade genética e ganhos de seleção em progênies de Myracrodruon urundeuva Fr. All. em sistema de multi-espécie. Nativa, Sinop, v. 5, p. 59/1-65, 2017.

REITZ, R.; KLEIN, R. M.; REIS, A. Projeto madeiras do Rio Grande do Sul. Sellowia, Itajaí, n. 34/35, p. 1-525, 1983.

RESENDE, M. D. V. Genética biométrica e estatística no melhoramento de plantas perenes. Brasília: Embrapa Informação Tecnológica, 2002. p. 975.

RESENDE, M. D. V. Melhoramento de essências florestais. In: BORÉM, A. Melhoramento de espécies cultivadas. Viçosa: UFV, 1999. p. 589 - 648.

RESENDE, M. D. V. Matemática e estatística na análise de Experimento e no melhoramento genético. Colombo: Embrapa Florestas, 2007. 362 p.

ROSADO, A. M.; ROSADO, T. B.; ALVES, A. A.; LAVIOLA, B. G.; BHERING, L. L. Seleção simultânea de clones de eucalipto de acordo com produtividade, estabilidade e adaptabilidade. Pesquisa Agropecuária Brasileira, Brasília, v. 47, n. 7, p. 964-971, 2012.

SANQUETTA, C. R.; DOLCI, M. C.; CORTE, A. P. D.; SANQUETTA, M. N. I.; PELISSARI, A. L. Estimação de volumes de Araucaria angustifolia (Bertol.) O. Kuntze por fatores de forma em classes diamétricas e modelos de regressão. Enciclopédia Biosfera, Goiânia, v. 13, n. 23, p. 588-597, 2016.

SANT'ANNA, C. S. ; SEBBENN, A. M. ; KLABUNDE, G. H. ; BITTENCOURT, R. ; NODARI, R. O. ; MANTOVANI, A ; REIS, M. S. . Realized pollen and seed dispersal within a continuous population of the dioecious coniferous Brazilian pine [Araucaria angustifolia (Bertol.) Kuntze]. Conservation Genetics, v. 14, p. 601-613, 2013.

SEBBENN, A. M.; FREITAS, S. A.; FREITAS, J. A Variação genética em cinco procedências de Araucaria angustifolia (Bert.) O. Ktze. no sul do estado de São Paulo. Revista do Instituto Florestal, São Paulo, v. 16, n. 2, p. 91-99, 2004 .

SEBBENN, A. M.; FREITAS, M. L. M.; ZANATTO, A. C. S.; MORAES, E. Seleção dentro de progênies de polinização aberta de Cariniana legalis Mart. O. Ktze (Lecythidaceae), visando à produção de sementes para recuperação ambiental. Revista do Instituto Florestal, v. 21, p. 27-37, 2009.

SEBBENN, A. M.; FREITAS, M. L. M.; ZANATO, A. C. S.; MORAES, E.; MORAES, M. A. Conservação ex situ e produção de sementes em banco de germoplasma de Balfourodendron riedelianum. Revista do Instituto Florestal, São Paulo, v. 19, n. 2, p. 101-112, 2007.

SEBBENN, A. M.; PONTINHA, A. A. S.; GIANOTTI, E.; KAGEYAMA, P. Y. Genetic variation in provenanceprogeny test of Araucaria angustifolia (Bert.) O. Ktze. in São Paulo, Brasil. Silvae Genetica, Frankfurt, v. 52, n. 5-6, p. 181-184, 2003. 
SEBbENN, A. M.; SIQUEIRA, A. C. M. F.; KAGEYAMA, P. Y.; MACHADO, J. A. R. Parâmetros genéticos na conservação da cabreúva (Myroxylon peruiferum L.F. Allemão). Scientia Forestalis, Piracicaba, v. 26, n. 53, p. 3138, 1998.

SHIMIZU, J. Y. Variação entre procedências de Araucária em Ribeirão Branco (SP) aos vinte e três anos de idade. Boletim de Pesquisa Florestal, Colombo, n. 38, p. 89-102, 1999.

SHIMIZU, J. Y.; JAEGER, P.; SOPCHAKI S. A. Variabilidade Genética em uma População Remanescente de Araucária no Parque Nacional do Iguaçu, Brasil. Boletim de Pesquisa Florestal, Colombo, n. 41, p. 18-36, 2000.

SOUSA, V. A. Population genetic studies in Araucaria angustifolia (Bert.) O. Ktze. 2001. 161 p. Thesis (PhD in Forestry) - Faculty of Forest Sciences and Forest Ecology, Institute of Forest Genetics and Forest Tree Breeding, Georg-August University of Göttingen, 2001.

SOUSA, V. A.; HATTEMER, H. H. Pollen dispersal and gene flow by pollen in Araucaria angustifolia. Australian Journal of Botany, Collingwood, v. 51, p. 309-317, 2003.

SOUSA, V. A.; ROBINSON, I. P.; HATTEMER, H. H. Variation and population structure at enzyme gene loci in Araucaria angustifolia (Bert.) O. Ktze. Silvae Genetica, Frankfurt, v. 53, n.1, p. 12-19, 2004.

STEFENON, V. M.; FINKELDEY, R. Genetic Structure of Araucaria angustifolia (Araucariaceae) Populations in Brazil : implications for the in situ conservation of genetic resources. Plant biology, New York, v. 9, n. 4, p. 516-525, 2007.

THOMAS, P. Araucaria angustifolia. IUCN Red List of Threatened Species. Version, 2013.

TOMAZELLO FILHO, M.; LATORRACA, J. V. F.; FISCHER, F. M.; MUÑIZ, G. I. B.; MELANDRI, J. L.; STASIAK, P. M.; TORRES, M. A.; PICCION, W. J.; HOFFMANN, H. A.; SILVA, L. D. Avaliação da Dispersão de Sementes de Pinus taeda L. pela Análise dos Anéis de Crescimento de Árvores de Regeneração Natural .Floresta e Ambiente, Seropédica, v. 24, e00040913, 2017.

VENCOVSKY, R. Genética quantitativa. In: PATERNIANI, E. (Coord.). Melhoramento do milho no Brasil. Campinas: Fundação Cargill, 1978. p. 122-201.

VENCOVSKY, R.; CHAVES, L. J.; CROSSA, J. Variance effective population size for dioecious species. Crop Science, Madison, v. 52, n. 1, p. 79-90, 2012.

WEI, R. P.; LINDGREN, D. Effective family number following selection with restrictions. Biometrics, Arlington, v. 52, n. 2, p. $198-208,1996$.

WHITE, T. L., ADAMS, W. T., AND NEALE, D. B. 2007. Forest genetics. Oxfordshire: CABI Publishing, 2007.

ZANON, M. L. B.; FINGER, C. G. Relação de variáveis meteorológicas com o crescimento das árvores de Araucaria angustifolia (Bertol.) Kuntze em povoamentos implantados. Ciência Florestal, Santa Maria, v. 20, n. 3, p. 467-476, 2010 .

ZANETTE, F.; DANNER, M. A.; CONSTANTINO, V.; WENDLING, I. Particularidades, biologia reprodutiva e hábitos de crescimento em plantas de Araucaria angustifolia. In: WENDLING, I; ZANETTE, F. (Org.). Araucária: particularidades, propagação e manejo de plantios. Brasilia: Embrapa, 2017. v. 1, p. 15-42.

Recebido em: 15/05/2017

Aceito em: 23/08/2017

Sci. For., Piracicaba, v. 46, n. 120, p. 519-531, dez. 2018

DOI: dx.doi.org/10.18671/scifor.v46n120.01 